\title{
Implementation of GUI for Class Attendance Using Face Detection and Recognition System
}

\author{
R. Karthikeyan, G. Nalinashini, R. Chithrakkannan, K. Mohanraj, B. UmaMaheswari
}

\begin{abstract}
In present years, improvements are made in teaching learning process by using digital systems. Class attendance is a indicator of student attentiveness, participation in academics and the interest the teacher creates in a particular subject. Quantitative analysis are carried out worldwide to understand the drop out percentage. Manual attendance may be a erroneous, time consuming process in which favour can also be done to a certain extent marking fake attendance. In this paper we propose a cost effective method to understand the attendance pattern of a student by use of face detection and recognition system where many features like lips, nose, iris etc. are distinct and data captured needs good computational analysis for recognition. In this paper we propose an automated class attendance system by creating a simple and user friendly GUI using LabVIEW software. The usefulness of this system will ensure error free attendance and will improve the teaching learning process by increase in lecture time and monitoring class attendance efficiently.
\end{abstract}

Keywords: Class Attendance, Face detection, Face Recognition, GUI.

\section{INTRODUCTION}

Class attendance in all academic institutions plays vital role in analysing the academic performance of any student. In recent days many random checks are done in the context of indentifying the defaulters. This process consumes more time for the teachers and reduces the lecture time. Use of many digital systems can reduce this time consuming process more drastically. The simple method of indentifying the presence of students in a classroom may be by a biometric application which is also a time consuming process and cannot be repeated for each period. Facial recognition techniques are simple and much improvement in recent days made the system robust and error free but the applications implemented are for security systems, criminal record identification, safety systems and more. The use of face recognition in class attendance may improve the performance of the student by thoroughly monitoring hourly attendance in each subject and his average overall retention in classroom. It also improves the efficiency in making an error free attendance log book for a faculty.

Revised Manuscript Received on October 15, 2019.

R.Karthikeyan, Assistant Professor, Dept of Instrumentation \& Control, Sri Sairam engineering college, Chennai, Tamilnadu, India.(Email: karthikeyan.ice@sairam.edu.in)

G.Nalinashini, Associate Professor, R.M.D. Engineering College, Kavaraipettai, Tamilnadu, India.(Email: gns.eie@rmd.ac.in)

R.Chithrakkannan, Associate Professor, Dept of Instrumentation \& Control, Sri Sairam engineering college, Chennai, Tamilnadu, India.(Email: chithrakkannan.ice@sairam.edu.in)

K.Mohanraj, Assistant Professor, Dept of Instrumentation \& Control, Sri Sairam engineering college, Chennai, Tamilnadu, India.(Email: mohanraj.ice@sairam.edu.in)

B.UmaMaheswari, Assistant Professor, Dept of Instrumentation \& Control, Sri Sairam engineering college, Chennai, Tamilnadu, India.(Email: maheshwari.ice@sairam.edu.in)
In this paper the practical needs of a cost effective and user friendly performance with higher accuracy is discussed. This method is a problem where the images or face recognition is considered to be a $2 \mathrm{D}$ information rather than a 3D information. A human face normally has distinct feature in lips, nose and iris. The Eigen face system can be made to execute an anonymous rate. The future work of this paper is to in addition to creating a GUI for attendance also to analyze and automate the psychology of a person towards a particular subject or teacher.

Many research works has been carried out in face recognition and detection. Among this Principal Component Analysis (PCA) is a fast, simple and effective method for face recognition. Arun Vyas has in his research work stated that a 2D array of values may be converted into simple image vectors and the entire simple image vectors of a particular feature is arranged into a set containing trained vectors then the Images are transformed into a dimensional space. The average of the feature extracted image is subtracted to compute the covariance. Another template method Support Vector Machine and Linear Discriminant analysis has their own limitations and advantages over PCA but still the template PCA method where the correlation measure, less noise, reduced memory and increased efficiency.

\section{PROPOSED SYSTEM}

The proposed system has four modules in which the image of a classroom which has a specific layout of the student is first captured and the image is processed, then the feature of the multiple face detected are indentified and stored in a binary data of Eigen values. The features considered here are the lips, nose and the iris of a particular student. The third module compares the data with the trained data 


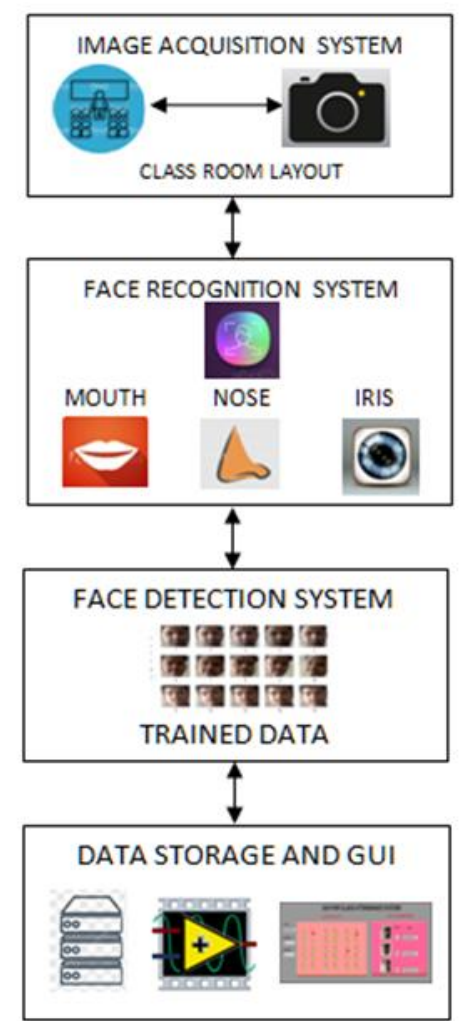

Fig. 1. Process flow diagram of the class attendance system.

which are initially acquired by taking individual images of each student before installing the module is training. In the last module the layout of the class room is matched with the available set of pre-defined data based and indicated in the GUI and provision is made to create a copy of the required student database either as an individual or as a group.

\section{A. Image Acquisition System}

The class room layout is created based on the roll number of the student. In this paper as an experiment a layout with 5 rows and 6 columns considering 30 students has been created. They are made to sit in a pattern according to their roll number but this is extended to a random seating arrangement after successful results. The image is captured facing the students from the top in such a manner all faces are completely captured and a hard wired switch is given so that the camera can capture images at the freedom of the teacher. The hardwire switch also initiates the software for face recognition. The image is captured at a resolution which can be processed by a laptop in this paper but for a robust application a high resolution camera should be used.

\section{B. Face Recognition System}

The image captured image is read in LABVIEW using vision toolbox. The matrix data values of the pixel values are given to an algorithm which based on a Viola Jones algorithm read the images acquired and crop the area of face into an individual image file in jpeg format of a lesser known resolution. Further each face is cropped into various features in this case three such features are lips, nose and the iris is taken separately.

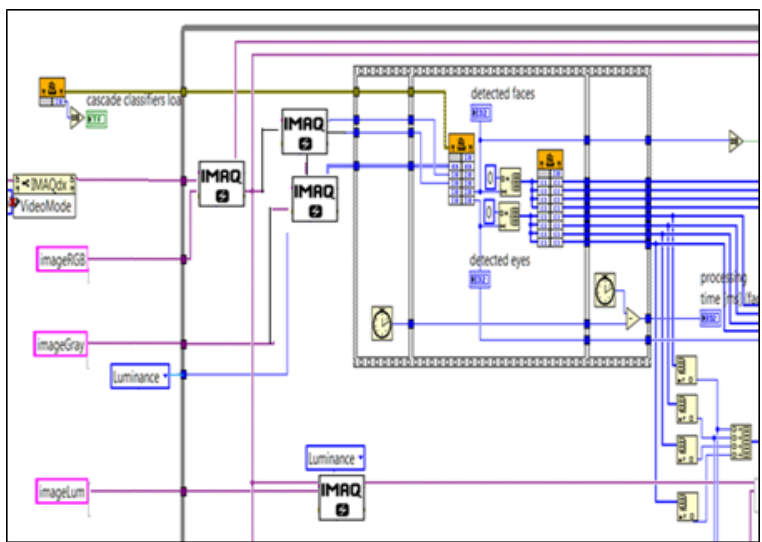

Fig. 2. Labview block diagram of face detection

Figure 2 shows the vision toolbox to capture image from the camera and converts image into binary values stored in a $2 \mathrm{D}$ array. The eigenface algorithm converts the 2D image data of each image with a known resolution to a $1 \mathrm{D}$ vector and several vectors creates a matrix of images. The mean value of the vector matrix is determined and is subtracted with vector of each image $1 \mathrm{D}$ vector and the average of it is formed into a new training matrix.

\section{Face Detection System}

The trained data of the features extracted by the different lips, nose and iris of the various students are stored after normalizing.

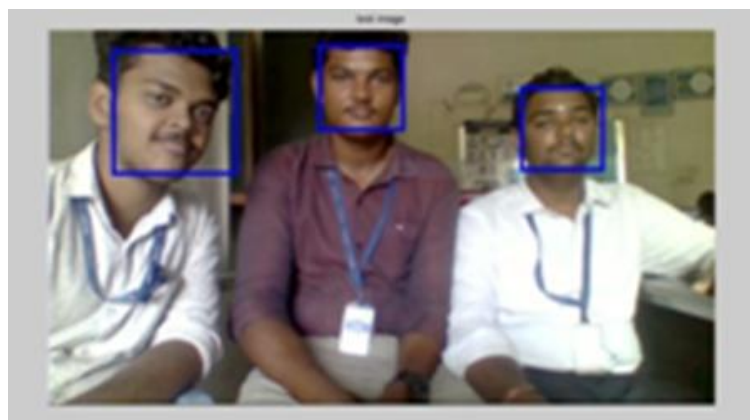

Figure 3. Cropping images to extract feature.

If distances between the samples image feature and training image feature are within the threshold. It is well thought-out belong to a particular student in the trained database otherwise it is measured to be unknown or a absent student. Whenever a person is effectively predictable the system marks the attendance in the GUI considering the database of the class layout the data of all recognized faces are stored in MS Excel.

\section{Data storage and GUI}

When the algorithm is executed it indentifies a match, the corresponding layout of the student is either indicated as red or green based on the students absence or presence respectively. It is updated in the MS Excel with their roll number on a particular date and time in addition by default it is marked with binary value zero which indicates that the student is absent. MS Excel provides a very efficient way of 
storing the data. Different GUI is created to easily understand the attendance system.

A first GUI is provided with a layout a marking the presence of a student in green and absence in red it also give the name, roll number and the image of the student absent on that particular day. The second GUI is created to give details of a particular student requested by the teacher form specified set of dates

The third GUI used to generate the list of student who has a specified percentage of presence for a teacher specified dates.

\section{RESULTS}

Using the database of 30 enrolled students which are stored in the folder. A sample image is captured and for experiment purpose stored as a jpeg file. The image has a resolution of $720 \times 526$ pixels. The speed of calculation varies with the resolution and also the accuracy of the algorithm increases with increase in resolution. However, speed of computation may not be a problem in this application since the attendance system is a non sensitive application where computational time is a vital parameter because the class attendance can be taken with the specified time duration of the class which may normally in hours.

Several limitations are needed to be taken into account they are illumination in the class room, the sitting poisture of the student such that the captured image contains all the students face upright such that the features may be detected.

From the data stored in the MS Excel the information of the classroom is displayed in the front panel of the GUI. Figure 4 shows the details of the class attendance system it indicates the present date, number of students present and number of student absent It also indicates in the layout which roll numbers are absent as well as shows the image of the student absent, name of the student and roll number of the student. From figure it is clear that roll numbers $6,23 \&$ 26 are absent on that particular day.

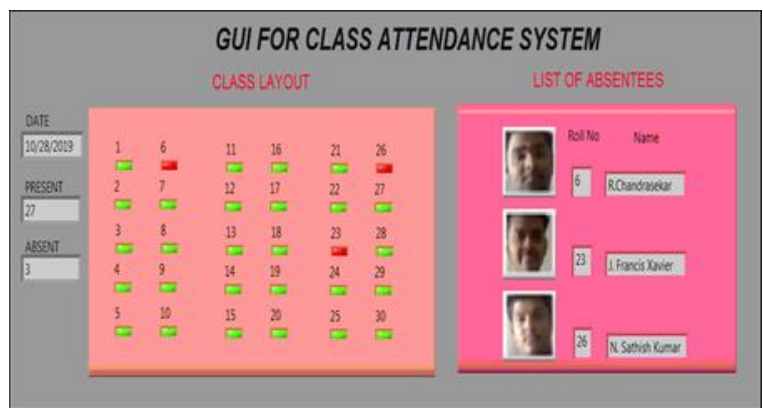

Fig. 4. Labview front panel diagram of face class attendance system

Figure 5, is a graphical panel which shows the details of a particular student which can be selected by the teacher, also provision are provided to enter from date and to date. The GUI gets the data stored from the MS Excel sheet for the particular name and displays the total days within the period, the total number days the student was present and total number days the student was absent within the selected days. Provision is given to print the data.

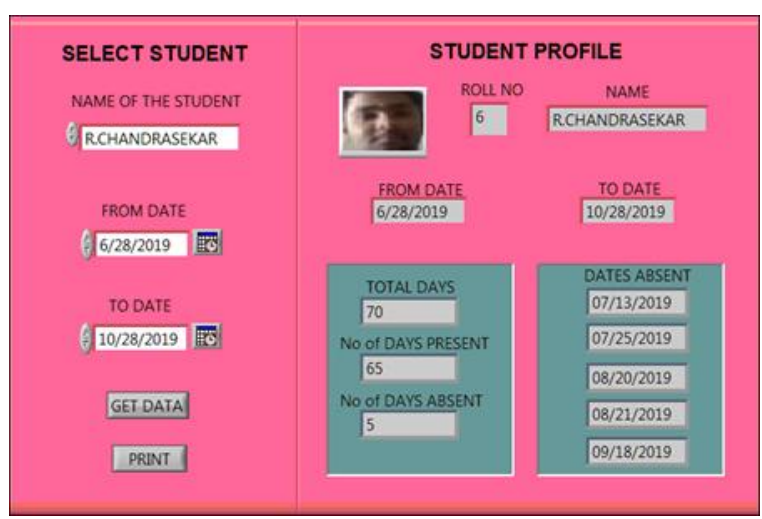

Fig. 5. Labview front panel diagram a particular student profile.

From the data stored the average attendance of a student the percentage attendance of a student can be calculated and it can be displayed on request. Figure 6, shows the list of students who are having attendance percentage of $>75$. Provision is given for selecting the percentage range from greater than 75 , less than 75 and less than 50 within the specified dates so that the teacher can easily counsel the student to be regular if attendance percentage is very lower than the prescribed limits. The front panel indicates the total days specified the list of students with their attendance percentage and the days they were present and day they were absent.

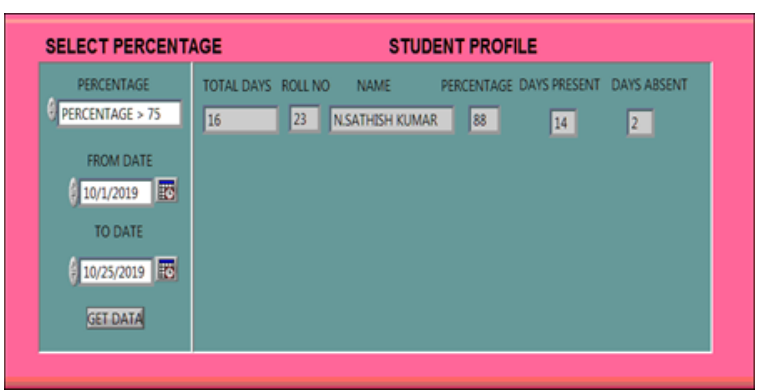

Fig. 6. Labview front panel diagram for a group of students who are having attendance percentage of $>75$.

Figure 7, show the list of students who has a attendance percentage of $<50$ with the total days specified the list of students with names and their attendance percentage, the days they were present and day they were absent. The percentage can be taken as a print out

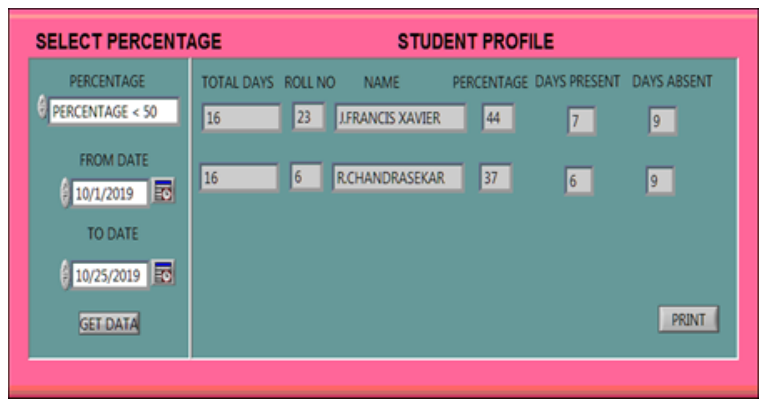

Fig. 7. Labview front panel diagram of a group of students who are having attendance percentage of $<50$. 
Figure 8, shows the output of the stored data in a MS Excel It can also be stored in any appropriate format based on the operating system used.

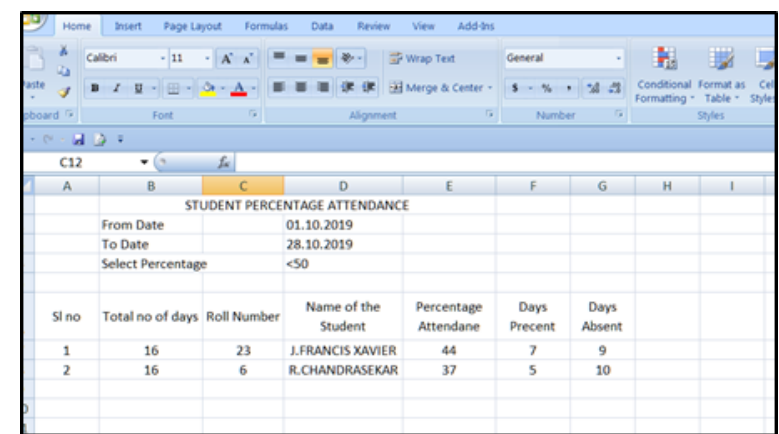

Fig. 8. MS Excel Output sheet for database storage and printing.

\section{CONCLUSION}

Using Labview for class automated attendance is a low solution for academic institutions. The automated systems reduce the errors in taking oral attendance and increases the lecture time by avoiding the teacher to take attendance. It can be useful to frequently check the student behavior and friends as the layout is mentioned where the student is seated for the particular period. Even though it has several limitations such as lighting, position of the student, difference in mood of the student expression while training and real time which may lead to error in detection care has been taken in positioning the camera and lighting system for the application.

Lighting during blackout has to be planned. The system studied with 30 students in a class can be extended for more number of classes when connected to a network. Future plans are to study, analyze the psychology of the student based on the yearlong data and can improve the teaching learning process.

\section{ACKNOWLEDGMENT}

It would have not possible to carry out this research without the support of our Management of Sri Sairam Engineering College, colleagues of Dept. of ICE and Mr. N. Balaji and Ms. T. Mangayarkarasi. We thank all of them for their enormous support, motivation, and resources

\section{REFERENCES}

1. Paul Viola and Michael J. Jones, "Robust Real-Time Face Detection," International Journal of Computer Vision, vol. 57, no. 2, pp. 137-154, May 2004.

2. Chetan.R, Rajesh Nayak, "Automated Attendance System Based on Facial Recognition International Journal for Research Trends and Innovation " Volume 2, Issue 6 | ISSN: 2456-3315

3. Hitesh Walia, Neelu Jain "Fingerprint Based Attendance System Using LabVIEW and GSM" International Journal of Innovative Research in Science, Engineering and Technology Vol. 5, Issue 7, July 2016

4. T.Thaj Mary Delsy, N.M.Nandhitha, Feasibility of spectral analysis techniques for disruption analysis in Aditya tokamak. International Journal of Engineering \& Technology, [S.1.], v. 7, n. 4, p. 3843-3846, dec. 2018

5. T.Thaj Mary Delsy, N.M.Nandhitha, "Spectral statistical analysis of low frequency coefficients from diagnostic signals depicting MHD disruptions", 978-1- 5090-49677/17/\$31.00 2017 IEEE,

6. Sandhya, P \& Thaj Mary Delsy T. (2016). Adaptive detection of pulmonary nodules in ct images by segmentation and classification using matlab. 8. 1198011985.

7. H. A. Mayank Agarwal, "Face Recognition Using Eigenfac e aproach," IRCSE, vol. 2, no. 4, pp. 17938201, August 2010.

8. Vinay Hermath, Ashwini Mayakar, "Face Recognition Using Eigen Faces and," IACSIT, vol. 2, no. 4, pp. 1793 8201, August 2010.

9. N.Balaji, S.Vijayalakshmi " FPGA Implementation of smart water quality monitoring system" International journal of innovative technology and exploring engineering, vol 8,11pg 3136-3139. 